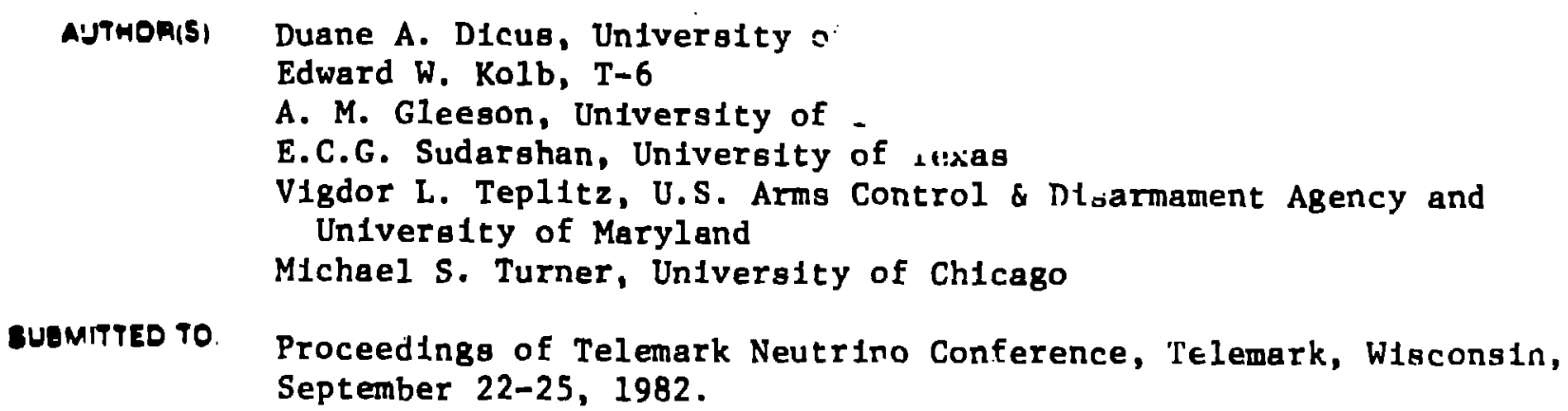




\title{
CORRECTIONS TO PRIMORDIAL NUCLEOSYNTHESIS
}

\author{
Duane A. Dicus, ${ }^{1}$ Edward W. Kolb ${ }^{2}$ A. M. Gleeson, ${ }^{1}$ \\ E.C.G. Sudarshan, ${ }^{1}$ Vigdor L. Teplitz, 3 and Michael S. Turner \\ ${ }^{1}$ Center for Particle Theory \\ Univissity of Texas \\ Austin, TX 78712 \\ "Theoretical Division \\ Los Alamos National Laboratory \\ Los Alamos, NM 87545

\begin{abstract}
The changes in primordial nucleosynthesis resulting from amall corrections to rates for weak processes that connect neutrons and protons are discussed. The weak rates are corrected by improved treatment of Coulomb and radiative corrections, and by inclusion of plasma effects. The calculations lead to a systematic decrease in the predicted ${ }^{4} \mathrm{He}$ abundance of about $\Delta Y=0.0025$. The relative changes in. other primordial abundances are also $1-2 \%$.
\end{abstract}


In this talk I will discuss improvements in the calculation of the primordial ${ }^{4} \mathrm{He}$ abuidance. The corrections are related to improved treatment of the weak $n \leftrightarrow p$ rates. I will compare the new values of the ${ }^{4} \mathrm{He}$ abundance to the standard calculation of Wagoner. 1 For details of our calculations, the reader is referred to our original paper. 2

The standerd hot big-bang model of the universe seems to provide a reliable framework for understanding the origin and evolution of our universe. ${ }^{3}$ One of the features of our present universe which is naturally explained in this model is the large abundance of "He. The success of primordial nucleosynthesis in predicting the large primordial abindance of ${ }^{4} \mathrm{He}$, and the relatively large abundance of $\mathrm{D}$, is usually considered to be the strongest evidence that the universe can be described by a Friedmann-Robertson-Walker cosmology at very early times. Because of this concordance, it is attrective to assume that the Friedmann-Robertson-Walker cosmology was applicable at the time of nucleosynthesis, and then demand that the resulting primordial abundances of the light elements be within bounds extrapolated from present observations.

This latter approach has been recently employed to limit the number of neutrinos at nucleosynthesis, and to put constraints on a number of exotic prticles, such as photinos, gravitinos, goldstinos, monopoles, quarks, stable leptons, ctc. The ${ }^{4} \mathrm{He}$ abundance has also been recently used to argue that the standard cosmological model does not fit the data. The same phenomenon has been called by others the "crowning achicvement of the standard model."

Because pribordial nucleosynthesis provides such a powerful prohe of the conditions in the universe at early times, it is importanf. Lo have prectse predictions of the primordial light element abundances, particularly that of ${ }^{4} \mathrm{He}$. In this talk, l will consider modifjcations to the calculation of the 'He abundance due to: 1) wse of an explicit numerical integration of the ratms for n-p transiljons ruther than iits lo the numerical rates; 2) correct treatment of Coulomb corrections; 3) inclusion of radintive corrections-hoth the usilal radialive corrections, and the rinje temperature and finlte density radiative corrections that depend on the presence of a plasma; 4) Incluston of the effect of the flanma on the mans oi the celectron; ant 5) 
heating of electron neutrinos in $e^{+} e^{-}$annihilation. We find that the above five effects result in a systematic decrease in the ${ }^{4} \mathrm{He}$ abundence of about 0.003 , or about a one percent relative decrease. (Addition of a light neutrino species leads to a $\Delta Y$ of $\Delta Y \simeq 0.01$.) There are similar $1-2 \%$ changes in the abundances of the other light elements which are produced ( $D,{ }^{3} \mathrm{He},{ }^{7} \mathrm{Li}$, etc.).

Although we find that the corrections to the weak rates are in general temperature dependent, we can get a rough estimate of the sensitivity of the primordial ${ }^{4} \mathrm{He}$ abundance, $Y$, on the weak rates by considering the change in $Y$ resulting from a change in the neutron balf-life. 5 For instance, a decrease in the neution half-life from 10.6 minutes to 10.4 minutes $\left(\Delta \lambda / \lambda \cong+0.02\right.$ where $\left.\lambda \equiv \tau_{1}^{-1}\right)$ results in a decrease ir. the ${ }^{4}$ He abundance of about 0.004 . T!nis decrease in the neutron half-ife is equivalent to an increase in $G_{F}$, the Fermi constant, hence a temperature independent increase in all the weak rates. This suggests that the dependence of $Y$ on $\Delta \lambda$ may be approximated as $\Delta Y \cong 0.2 \Delta \lambda / \lambda$.

It should be emphasized that the corrections $1-5$ abuve are universal in the sense that they must be applied regardless of the values of the neutron lifetine, the number of neutrinos, or the ratio of bacyons to photons.

The six weak reactions which interconvert neutrons and proton's are $n \leftrightarrow p e \bar{v}$, nè $\leftrightarrow p \bar{v}$, and $n u \leftrightarrow$ pe. The rates for these processes were first calculated in the context of the early tniverse by Alp'ser, Follin, and Herman, 5 and we wiil adopt their notation (see also ref. 1). The rates depend on $Q=m_{n}-m_{p}$, the photon temperature $T$, the neutrino temperature $T_{v}$, and the electron energy $F_{e}$. The rates depend on these quantities through the dimensionless variahlea $\Sigma=m_{e} / T$, $z_{v}=m_{e} / T_{v}, a=Q / m_{e}$, and $\varepsilon=E_{e} / m_{e}$. The weak rates may be written in terms of these variables. For instance, the race for neutron decay is

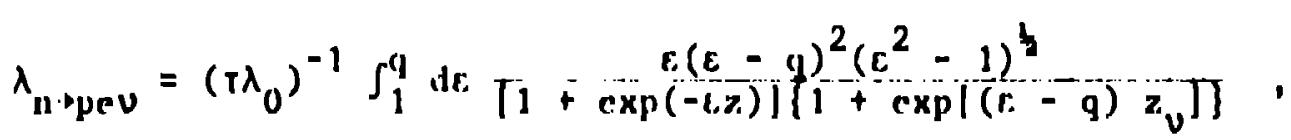


where $\tau$ in the neutron lifetime and $\lambda_{0}$ is defined as $\lambda_{0} \equiv \int_{1}^{q} d \varepsilon$ $\varepsilon(\varepsilon-q)^{2}\left(\varepsilon^{2}-1\right)^{\frac{1}{2}}=1.63615$. The factor $\left(\tau \lambda_{0}\right)^{-1}$ fixes the nuclear natrix element to be used.

In the computer code developed by Wagoner, ${ }^{1}$ the rates $(2.7)$ are $\mathrm{fit}$ by an analytic function of the photon temperature. The fit to $\lambda_{n}$ is good to an accuracy of about $1.4 \%$ over the temperature range $T_{9}=100$ to $\mathrm{T}_{9}=0.3\left(\mathrm{~T}_{9}\right.$ is the temperature in units of $\left.10^{9} \mathrm{~K}\right)$. The fit to $\lambda_{p}$ is good to about $1.4 \%$ over a range $T_{9}=100$ to $T_{9}=3$. This fit is rather remarkable considering that $\lambda_{n}$ and $\lambda_{p}$ change by more than seven orders of magnitude over the above temperature range. However, based on our approximate formula for $\Delta Y$ given in Eq. (1.1), a $1.4 \%$ error in the weak rates lead to a potential $\Delta Y$ of $|\Delta Y|=(0.2)(1.4 \%) \cong .003$.

We have modified Wagoner's code to evaluatc the rates numerically every time step. We evaluate the integrals to an accuracy of better than $0.005 \%$, which shouid introduce an error in $\Delta Y$ of $|\Delta Y| \lesssim 0.0001$. The change in $Y$ is given in Table $I$.

Our next change is to correctly treat the Coulomb corrections. Coulomb corrections are taken into account by multiplying the phase space density for $n \leftrightarrow$ pev and $n \cup \leftrightarrow$ pe by $F_{+}$, where $F_{+}$is the Fermi function given in terms of the electron velocity, $\beta$, as

$$
F_{+}(\beta) \cong \frac{2 \pi \alpha / \beta}{1-e^{-2 \pi \alpha / \beta}}
$$

It is also necessary to include $F_{+}$in the phase space for $\lambda_{0}$, which changes $\lambda_{0}$ from 1.63615 to about 1.69. Wagoner accounted for Coulomb corrections by decreasing $\lambda_{0}$ by $2 \%$. The effect of correctly treating the Coulomb corrections is shown in Table $I$.

Our next correction is to include the radiative corrections. The Radiative corrections have been considered by many authors, ${ }^{0}$ the ettect is to multiply the phase space for all processes by $11+\frac{\alpha}{2 \pi}$ $C(\beta, y))$ where $\beta$ is the electron's velocity, and $y$ is the neutrino energy divided by $m_{e}$ ( $y$ ls different for the different weak piocesses). The function $C$ is given iy 


$$
\begin{aligned}
C(\beta, y) \cong & 40+4(R-1)(y / 3 \varepsilon-3 / 2+\ln 2 y) \\
& +R\left[2\left(1+\beta^{2}\right)+y^{2} / 6 \varepsilon^{2}-4 \beta R\right] \\
& -4\left[2+11 \beta+25 \beta^{2}+30 \beta^{3}+20 \beta^{4}+8 \beta^{5}\right] /(1+\beta)^{\epsilon} .
\end{aligned}
$$

In Eq. (3) the last term in the brackets is from expansions of Spence functions, and $R$ is defined to be

$$
R \equiv \beta^{-1} \tanh ^{-1} \beta .
$$

Including radiative corrections changes $\lambda_{0}$ to 1.75321 . Wagoner ignored radiative corrections. The effect of including the radiative corrections is given in Table I.

We next correct the rates by doing the radiative corrections at finite temperature. These corrections were also done independently by Cambier, Primack, and Sher. ${ }^{7}$ The real-time formalism ${ }^{8}$ is particularly useful for this purpose, since there is a clean separation between temperature dependent and the temperature independent parts of the radiative corrections.

The finite cemperature radiative corrections involve double integrals which must be done numerically. The integrals are too tedious to write here; they are given elsowhere. ${ }^{2,7}$ The finite +emperature rudiative corrections cause the rates to be multiplied by al additional factor of the frim

$$
1+\frac{\alpha}{\pi} C_{i}^{\prime}(T)
$$

where the $C_{i}^{\prime}$ differ for different processes.

It turns out that the finite temperature corrections depend strongly on the temperature and on the individual reaction considered. It is also turns out that the rate for neutron decay receives the largest correction. This large correction is mostly due to the effect of a photon in the initial state: $y_{n} \rightarrow$ pev. The effect ol the photon can be thought of as an effective increase in the $Q$ value for $n \rightarrow$ pev, $Q \rightarrow Q+\mathbb{W}$, where $w$ is the energy of the photon, $\langle\omega\rangle \cong 2.7 T$. Since the neutron decny rate is a more sensitive 
function of $Q$ than the $2 \rightarrow 2$ weak processes, this particular consequence of finite temperature is larger for $\lambda_{n \rightarrow p e v}$. However, at the time of the freeze out of the neutron-proton ratio, $\lambda_{n \rightarrow p e v}$ makes only a small contribution to the total rate. The effect of the finite temperature radiative corrections is shown in Table $I$.

The next effect we consider is the mass shift of the electron due to finite temperature effects. The electron mass at finite temperature is. found by calculating the electron self-energy diagram with the temperature dependent real time propagators. If one defines mass renormalization at zero temperature by the addition of a counter term $\delta m=-R e \Sigma\left(p^{2}=m_{e}^{2}\right)$ with $\Sigma(p)$ the electron self-energy, then finite temperature effects add to the electron mass a term $\delta m_{T}$ defined hug

$$
\delta m_{T}=\delta m+\operatorname{Re} \Sigma_{T}\left(p^{2}=m_{e}^{2}\right),
$$

where $\Sigma_{T}$ is the electron self-energy evaluated with the finite temperature photon and electron propagators. This results in a temperature dependent effective electron mass, $m_{T}$, of the form ${ }^{9}$

$$
\begin{aligned}
m_{T} & =m_{e}+\delta m_{T} \\
& =m_{e}+B \alpha T^{2} / m_{e}, \quad\left(\alpha T^{2} / m_{e}^{2} \leq 1\right),
\end{aligned}
$$

where $m_{e}$ is the zero temperature electron mass $(0.511 \mathrm{MeV})$, and $B$ is a slowly varying function of the temperature with a value between 1 and 2 .

It is easy to inciude this correction in the rates by the substitution of $m_{r}$ for $m_{e}$ in the rates and by multiplying all the rates by $\left(m_{T} / m_{e}\right)^{5}$, as the cancellation of the $m_{e}^{5}$ from the numerator, with the $m_{e}^{5}$ from $\lambda_{0}$ is true only at zero temperature. (We did not explicitly include the factor of $\mathrm{in}_{e}^{5}$ in the definitions of the rates and in the definition of $\lambda_{0}$.)

The modification of the election mass also has the effect of changing the relationship between the neutrino und photon temparatures. The neutrino temperature differs from the photon temperature because, to a good approximation, the neutrinos are decoupled when the culropy in the $f^{+} c^{-}$gas is released us $e^{+}$annihtlace. Therefore, the entropy released heals the photons, but not the neutrinos. Below, we discuss the validity of the 
approximation that the neutrinos are not heated by $e^{+} e^{-}$annibilations, and the effect on $Y$ of relaxing this assumption.)

An additional effect of the change in the electron mass that leads to an even smaller effect on $\Delta Y$, is that as the electron mass changes, the electron's contribution to the total energy density, and hence to the expansion rate, changes.

The changes in the primordial 4 He abundance due to finite temperature effects on the electron mass is given in Table $I$.

A final small effect we have considered is neutrino heating due to $e^{+} e^{-}$annihilation. Part of the "early Universe" lore is that neutrinos decouple at a temperature $T_{d} \cong 1 \mathrm{MeV}$ and therefore do not share in the entropy release from $e^{ \pm}$annihilations which occur at temperatures $\leq 0.5 \mathrm{MeV}$. As a consequence, neutrinos are predicted to have a lower temperature today than the photcns, $T_{v}=(\dot{4} / 11)^{1 / 3} T_{\gamma}$. The assumption of complete neutrino decoupling is explicitly incorporated into Wagoner's code. However, since the weak rates are extremely sensitive to the neutrino temperature, we have investigated the validity of this assumption. We have found thit the usual approximation of $\mathrm{T}_{v}=(4 / \mathrm{H})^{1 / 3} \mathrm{~T}_{\gamma}$ is valid to $2 \times 10^{-3}$ for $v_{e}$, and $9 \times 10^{-4}$ for $v_{\mu}$ and $v_{\tau}$.

The slightly wurmer neutrinos change the weak rates, and change the contribution to the total energy density due to neutrinos. The effects go in opposite directions, and tend to cancel.

The size of the effect is small. It is listed in Table I.

The changes in the predicted ${ }^{4} \mathrm{He}$ mass fraction due to all the effects discussed here are summarized in Table I. For a wide range of input parameters $\left(\tau_{\frac{1}{2}}=10.1-11.1 \mathrm{~min}, N_{v}=2-10\right.$, and $\left.n=3 \times 10^{-11}-3 \times 10^{-9}\right)$, the sum of all these corrections results in an approximately constant, systr. malic decrease in $Y, \Delta Y \cong-0.0025$ ( 1\% relative change). The changes in the prcdicted abundances of the other light elements (D, ${ }^{3} \mathrm{He}$, and ${ }^{7} \mathrm{Li}$ ) are also in the range of a few percent. However, their present abundances ore known to much less precision than the present "He abundance, typically to only within a factor of 2 . In Fig. 2 we show the predicted primordial ${ }^{4}$ He abundance with and withrit the corrections we have discussed in this paper, as a function of $\eta$ for $\tau_{\frac{1}{2}}=10.6 \mathrm{~min}$ and $N_{v}=2,3$, and 4 .

The net $\Delta Y$ we find is about the size of Wagoner's estimated uncertainty, ancl fust olfghty less then the uncertainty due to a 1 o change ( $\pm 0.16 \mathrm{~min}$ ) 
in $\tau_{1}$. Nevertheless, it is a systematic decrease in the predicted primordial abundance of ${ }^{4} \mathrm{He}$. Within a few years experiments with confined neutrons should significantly improve the determination of $\tau_{\frac{1}{2}}$. Recent studies of extragalactic, very metal-poor objonts (in which the stellar contribution to the ${ }^{4} \mathrm{He}$ abundance should be small) have led to more accurate and reliable determination of the primordial mass fraction of $4 \mathrm{He} .{ }^{10} \mathrm{~A}$ recent result typical of these studies is $Y_{p}=0.24 \pm 0.01 .10$ As more objects are studied the unceratinty in $Y_{p}$ should continue to decrease. It has been argued on the basis of the abundances of $D$ and ${ }^{3}$ He that $\eta$ must be greater than $(2-3) \times 10^{-10} .11$ For $N_{v}=3$ the standard model did predict $Y_{p} \gtrsim 0.240-0.246$ - leaving little room for concordance. It now predicts $y_{p} \geq 0.237-0.243$ - easing the situation a bit. In any case, since primordial nucleosynthesis is the most vigoious test of the standard, hot bif bang model, and is also our most powerful probe of the early universe, it is important to continue to sharpen and to cecxamine its predictions as the uncertainties in the input parameters decrease.

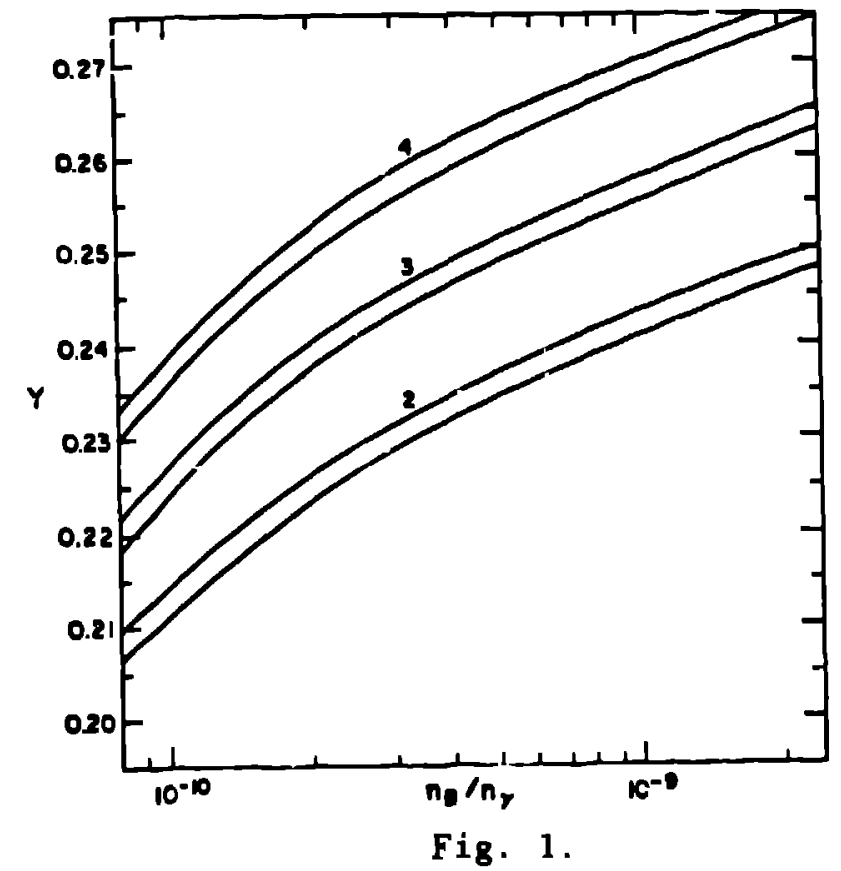

The primordial ${ }^{4}$ He mass fraction as a function of the baryon-to-photon ratio with 2,3 , and 4 light neutrino families assuming a neutron half-life of 10.61 minutes. The upper curve in each set wows the result of Wagoner's calcualtion, and the lower curve shows the results of including all the corrections 10 the weak rates. 


\section{TABLE I}

SENSITIVITY OF THE PRIMORDIAL ${ }^{4} \mathrm{He}$ ABUNDANCE TO THE CORRECTIONS DISCUSSED IN THF, TEXT

(The values quoted are for $n_{B} / n_{y}=3 \times 10^{-10}, 3$ light neutrinos, and $\tau_{\frac{1}{2}}=10.6$ minutes.)

Wagoner $1973 \quad Y_{0}=0.2456$

1. Wagoner $73 \quad Y_{1}=0.2443 \quad Y_{0}-Y_{1}=0.0013$ evaluation of rates

2. Above corrections plus

$Y_{2}=0.2434 \quad Y_{0}-Y_{2}=0.0022 \quad Y_{1}-Y_{2}=0.0009$

Coulomb

corrections

3. Above corrections plus $Y_{3}=0.2439 \quad Y_{0}-Y_{3}=0.0017 \quad Y_{2}-Y_{3}=-0.0005$ zero temperature radiative corrections

4. Above corrections plus finite temperature radiative corrections

5. Above cor-
rections plus corrections to electron mass

$$
Y_{5}=0.2436 \quad Y_{0}-Y_{5}=0.0020 \quad Y_{4}-Y_{5}=-0.0001
$$

6. Above corrẹctions plus $Y_{4}=0.2435 \quad Y_{0}-Y_{4}=0.0021 \quad Y_{3}-Y_{4}=0.0004$ $e^{+}$heating $Y_{6}=0.2434 \quad Y_{0}-Y_{6}=0.0022 \quad Y_{5}-Y_{6}=0.0002$ of $v_{e}$ 


\section{REFERENCES}

1. The version of the code we compare to is described in $R$. $V$. Wagoner, Astrophys. J. 1979, 343 (1973).

2. D. A. Dicus, E. W. Kolb, A. M. Gleeson, E. C. G. Sudarshan, V. L. Teplitz, and M. S. Turner, Phys. Rev. D., to be published.

3. For a review of the standard big-bang cosmology, see, e.g., S. Weinberg, Gravitation and Cosmology (Wiley, New York, 1972), Chap. 15.

4. Needless to say, we do not agree with this interpretation.

5. R. A. Alpher, J. W. Follin, and R. C. Herman, Phys. Rev. 92, 1347 (1953).

6. E. S. Abers, D. A. Dicus, R. E. Norton, and H. R. Quinn, Phys . Rev. 167, 1461 (1968); D. A. Dicus and R. E. Norton, Phys. Rev. D1, $1 \overline{360}$ (1970); M. A. B. Bég, J. Eernstein, and A. Sirlin, Phys. Rev. D6, 2597 (1972); T. W. Appelquist. J. R. Primack, and H. R. Quinn, Phys. Rev. DÉ, 2998 (1972); and A. Sirlin, Rev. Mcd. Phys. 50, 573 (1978).

7. J.-L. Cambier, J. R. Primack, and M. Sher, unpublished.

8. See, e.g., E. S. Fradkin, Zh. Eksp. Teor. Fiz. 36, 1286 (1961) [Sov. Phys. JETP 9, 912 (1959)]; V. N. Tsytovich, Zh. Eksp. Teor. Fiz. 40, 175 (1961) (Sov. Phys. JETP 13, 1249 (1961)]; A. Bechler, Ann. of Phys. 135, 19 (1981).

9. S. I. Glashow, E. C. G. Sudarshan, and A. Yildiz, unpublished; also see G. Peressutti and B.-S. Skagerstam, Phys. Lett. 110B, 406 (1982).

10. Some recent ${ }^{4}$ He determinations for metal-ponr objects include: $H$. B. French, Ap. J. 240, 41 (1980); J. Lequeuz, M. Peimbert, J. F. Rays, A. Serrano, and S. Torres-Piembert, Astro. Astrophys. Co, 155 (1979); D. L. Talent, Ph.D. Thesis, Rice University (1980); J. F. Rays, M. Piembert, and S. Torres-Piembert, Ap. j. $\underline{255}, 1$ (1982).

11. J. Yang, M. S. Turner, G. Steigmar, D. N. Schramm, and K. A. Olive, in preparation (1982). 